\title{
Defect sensitivity in additively manufactured aluminium al- loys: contribution of CAD artificial defects
}

\author{
Matthieu Bonneric ${ }^{1,2, *}$, Charles Brugger ${ }^{2}$, and Nicolas Saintier ${ }^{2}$ \\ ${ }^{1}$ IRT Saint Exupery, 3 rue Tarfaya - CS 43436, 31405 Toulouse cedex 4, FRANCE \\ ${ }^{2}$ I2M, Arts et Métiers ParisTech, CNRS UMR 5295, Université Bordeaux 1, FRANCE
}

\begin{abstract}
The prediction of the fatigue resistance of additively manufactured parts is a current issue for the materials and process qualification in aerospace industry. Despite a continuous improvement of AM process, the presence of defects cannot yet be completely avoided, and the latter are still one of the main causes of fatigue damage in AM materials. In this framework, the present work focused on the influence of defects on the uniaxial fatigue behavior of AlSi7Mg0.6 alloy produced by Selective Laser Melting (SLM). Uniaxial fatigue tests have been performed. Fatigue specimens were subjected to a T6 treatment, and then machined in order to avoid the influence of surface roughness. Besides, for some specimens, artificial defects were directly introduced through CAD. The introduction of artificial defects, whose sizes and positions are precisely controlled, aims to provide a proper assessment of defect sensitivity. X-ray tomography was used to characterize both natural and artificial defects. Finite-element calculations of the local stress fields in the vicinity of defects were conducted, accounting for the real defect geometries obtained with CT scans. The application of a non-local multiaxial fatigue criterion then allowed to analyze defect criticity.
\end{abstract}

\section{Introduction}

Over the past years, the production of Al-Si alloys by Selective Laser Melting (SLM) has been broadly studied, attempting to understand the relations between process parameters, microstructure, and mechanical properties [1]. Although a serious progress has been made to improve the process, the parts obtained by SLM still contain defects that are impossible to eliminate, which are found to be the main cause of fatigue damage [2-4]. Therefore, studying the defect sensitivity of the fatigue resistance is an important matter for these alloys.

The fatigue behavior of defective materials being a common issue for conventional processes, different approaches already exist in the literature to account for the presence of defects in fatigue strength prediction. It is usually assumed that the size of the largest surface or subsurface defect is the key feature that controls the fatigue strength [5]. In several works, defects are considered as an initial crack and fatigue strength is assessed from the defect size using a fracture mechanics framework [5-7], considering that the fatigue strength is the minimum stress above which a fatigue crack is not only capable of initiating, but also of

\footnotetext{
*e-mail: matthieu.bonneric@ irt-saintexupery.com
} 
propagating over the first microstructural barriers. In particular, this method was already employed to model the fatigue strength of cast aluminium alloys [8] and of an AlSi10Mg alloy obtained by SLM [4]. However, this kind of approach presents some limitations, especially because of the so-called short crack problem for which Linear Elastic Fracture Mechanics (LEFM) is not applicable [9], and is not convenient for multiaxial conditions. Another approach consists in treating the defect as a notch, and considering the stress distributions in its neighborhood to apply a stress-based fatigue criterion. For such a case, the stress gradient at the tip of the notch is an important matter, since it is directly related to the ability of the crack to propagate once initiation has occurred. The fatigue criterion can then be modified to account for it, either by averaging the stress over a physical dimension (point, length or volume) $[10,11]$, or by the explicit introduction of the stress field gradient in the criterion [12].

The aim of this study is to investigate the defect sensitivity of an AlSi7Mg0.6 alloy obtained by SLM. To do so, fatigue tests results are compared to finite element calculations accounting for the real defect geometries obtained by X-ray tomography, and from which a non-local fatigue criterion is applied [13]. Besides, artificial defects are also used by introducing holes directly into the specimen CAD files. Such a procedure allows not only to limit the scattering of the experimental results, but also to investigate the influence of defect position independently from defect size.

\section{Material and Methods}

\subsection{Material}

Samples for fatigue testing were obtained by SLM using the AlSi7Mg0.6 aluminium alloy. They were produced on a SLM $280 \mathrm{HL}$ powder bed machine, adopting the process parameters recommended by the manufacturer for Al-Si alloys (table 1), and after drying the powders under inert environment at $150^{\circ} \mathrm{C}$ during $12 \mathrm{~h}$. After manufacturing, samples were subjected to a T6 heat-treatment, consisting in a solution treatment during $2 \mathrm{~h}$ at $535^{\circ} \mathrm{C}$, followed by a water quenching and an artificial aging during $4 \mathrm{~h}$ at $170{ }^{\circ} \mathrm{C}$. Observations of the resulting microstructure, not detailed here, showed grains elongated in the building direction, of size $10 \mu \mathrm{m}$ when considering transverse directions. Si precipitates were also observed on grain boundaries, as well as the complete elimination of the dendritic structure due to T6 treatment.

Table 1: Process parameters recommended for Al-Si alloys

\begin{tabular}{ccccccc}
\hline $\begin{array}{c}\text { Layer } \\
\text { thickness }\end{array}$ & $\begin{array}{c}\text { Laser } \\
\text { power }\end{array}$ & $\begin{array}{c}\text { Scan } \\
\text { velocity }\end{array}$ & $\begin{array}{c}\text { Hatch } \\
\text { spacing }\end{array}$ & $\begin{array}{c}\text { Scanning } \\
\text { strategy }\end{array}$ & $\begin{array}{c}\text { Base plate } \\
\text { heating }\end{array}$ & Atmosphere \\
\hline $30 \mu \mathrm{m}$ & $350 \mathrm{~W}$ & $1650 \mathrm{~mm} / \mathrm{s}$ & $130 \mu \mathrm{m}$ & Stripes & $150^{\circ} \mathrm{C}$ & Argon \\
\hline
\end{tabular}

\subsection{Fatigue testing}

Tension/tension fatigue tests were performed on cylindrical specimens of axis parallel to the building direction, whose dimensions are given in figure 1. Two sets of specimens were produced: the first set, denoted as NDP, consists in specimens containing only natural defects resulting from the SLM process (Natural Defect Population), whereas the second one AD corresponds to specimens with Artificial Defects. It should be noted that all the specimens were 


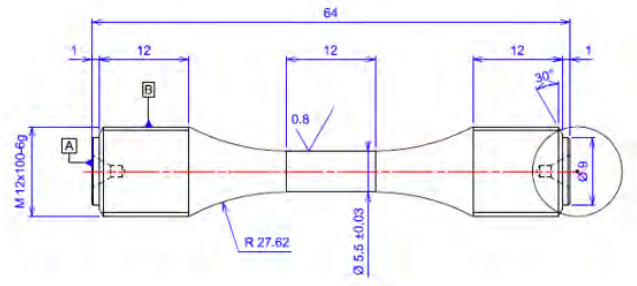

Figure 1: Geometry of the machined specimens used for HCF fatigue tests

machined in order to avoid the influence of surface roughness. HCF tests were carried out on a Zwick resonant machine with a $R=0.1$ load ratio, at room temperature in air. The stop criterion was a frequency drop of $0.5 \mathrm{~Hz}$, corresponding to a fatigue crack of approximately $1.5 \mathrm{~mm}$ in depth, or a maximum number of cycles of $2 \times 10^{6}$.

\subsection{Natural Defect Population}

The gage length of fatigue specimens was observed prior to fatigue testing using X-ray tomography with a spatial resolution of $4.7 \mu \mathrm{m}$, with the aim of characterizing the defects likely to be responsible for fatigue damage. Central part of three fatigue specimens were fully imaged, which represent a total data set of 268115 defects. As the fatigue behavior is mostly driven by the larger defect population, a peak-over threshold (POT) strategy was chosen to characterize this particular population, as proposed in [14]. To do so, only the largest defects of size $\sqrt{\text { area }}$ higher than a threshold were considered. Figure 2 provides the experimental defect size distribution so obtained from all the specimens, by choosing a threshold of $74 \mu \mathrm{m}$. It can be seen that experimental data can be fitted with a General Extreme Value (GEV) distribution of equation $P_{\text {cum }}(x)=e^{-\left(1+\xi \frac{x-\mu}{\lambda}\right)^{-\frac{1}{\xi}}}$, with $\xi \neq 0$. It is worth noting that the mean defect size is approximately $100 \mu \mathrm{m}$, corresponding to ten times the grain size, which is one of the most influential microstructural feature impacting on the fatigue behavior for cast $\mathrm{Al}-\mathrm{Si}$ alloys $[8,15,16]$.

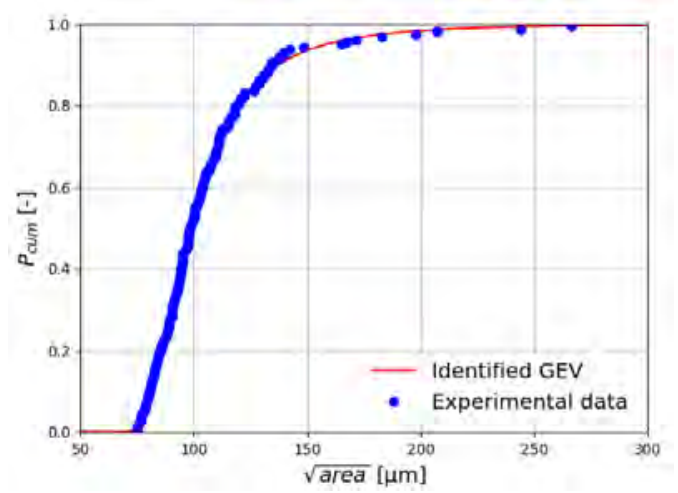

Figure 2: Distribution of the largest defects obtained from X-ray tomography observations of 3 fatigue specimens 


\subsection{Artificial Defects}

In order to both limit the scattering related to defect sizes and positions and investigate a wider range of defect sizes, artificial defects were generated in some fatigue specimens. Ellipseshaped holes of diameter $\phi=600 \mu \mathrm{m}$ and height $h=300 \mu \mathrm{m}$ were introduced directly into CAD files, so that after machining defects are $450 \mu \mathrm{m}$ from the surface. This last distance $d$ was chosen so that the ratio $\sqrt{\text { area }} / d$ was more than 0.8 , with the aim of generating subsurface defects according to Murakami's definition [5]. One can note that these defects are much larger compared to natural ones described in 2.3. Figure 3 provides views from $\mathrm{X}$-ray tomography observations of such a defect. Even if the shape of the defect cannot be entirely controlled and differs from $\mathrm{CAD}$, the actual defects are found reproducible in terms of size, $\sqrt{\text { area }}$ being $630 \mu \mathrm{m} \pm 4 \%$ for the different defects considered here. However, due to the uncertainty related to the machining, the distance between the center of the defect and the surface might vary from $20 \%$ from the targeted $450 \mu \mathrm{m}$. It should be mentioned that unlike for milling or EDM procedures that only allow to produce surface defects, the present procedure makes it possible to generate subsurface or internal defects.

To this day, the fatigue tests on the specimens containing artificial defects have not been performed yet. Thus, only finite-element results are presented in what follows for this kind of defect.

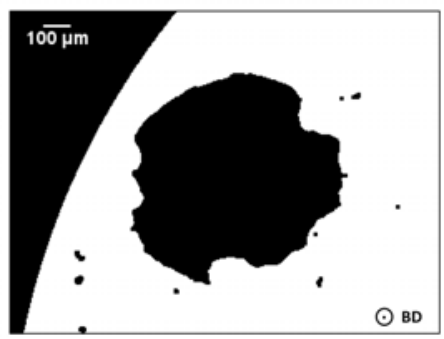

(a) Projection on a transverse plane

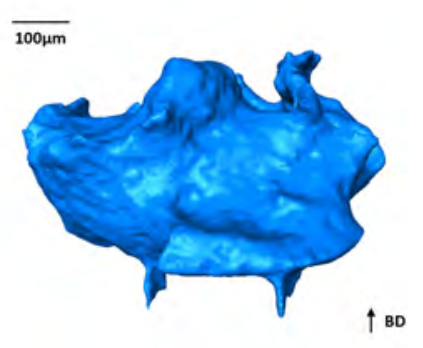

(b) $3 \mathrm{D}$ view

Figure 3: Views from X-ray tomography measurements of an artificial defect introduced in a fatigue specimen. Building direction (BD) matches the loading direction.

\subsection{Numerical simulations}

Fatigue cracks being likely to initiate from surface or subsurface defects for NDP fatigue specimens, a particular attention was paid to the largest natural defects observed with X-ray tomography in the near-surface volume (figure 4a). Defects from the larger defect population determined in 2.3 whose distance to the surface was less than $100 \mu \mathrm{m}$ were selected, based on the analysis of the critical defects observed on the fracture surfaces presented in 3.1. Indeed, fractography analysis revealed that the distance from the surface was always less than $100 \mu \mathrm{m}$ for the critical defects. Each defect surface was then meshed (figure $4 \mathrm{~b}$ ), prior to meshing a volume corresponding to a small portion of the fatigue specimen containing the defect (figure 4c). The meshed defects were manually positioned so that both distances and orientations with respect to the surface coincide with observations. The same procedure was also used for artificial defects. 


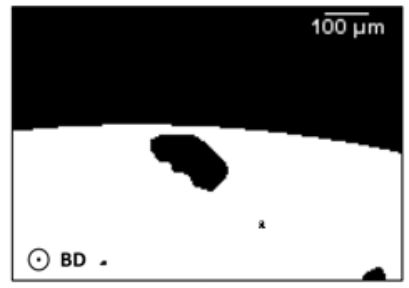

(a) CT scan observation of a subsurface natural defect - projection on a transverse plane

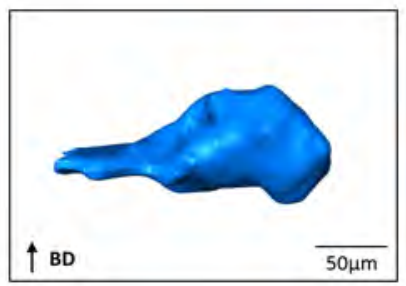

(b) Meshing of the defect surface

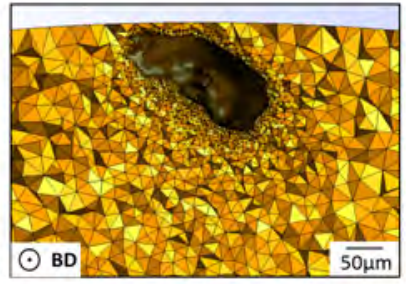

(c) Meshing of a portion of the specimen containing the defect - transverse cut

Figure 4: Procedure used to mesh a volume containing a natural defect, accounting for both the real defect geometry and position

Calculations were then performed using the FE code ZEBULON, applying a cyclic axial stress corresponding to the experimental fatigue resistance, with a $R=0.1$ load ratio. Quadratic elements were used, and the material behavior was modeled with a linear elastic isotropic law in a first approach $(E=70 \mathrm{GPa}, v=0.33)$. It should be mentioned that elastoplastic computations are currently under progress. In order to quantify defect criticity, Crossland criterion was used [13]: the fatigue strength is reached when the Crossland equivalent stress $\sigma_{C R}$ equals a threshold parameter $\beta$, with $\sigma_{C R}$ a combination of the amplitude of the deviatoric stress tensor second invariant $\tau_{\text {oct, } a}$ and the maximum hydrostatic stress $J_{1, \max }$ :

$$
\sigma_{C R}=\tau_{o c t, a}+\alpha J_{1, \max }
$$

In the above expression, the calculation of $\tau_{o c t, a}$ is obtained by a double maximization over the loading period:

$$
\tau_{o c t, a}=\frac{1}{2 \sqrt{2}} \max _{t_{i} \in T}\left\{\max _{t_{j} \in T} \sqrt{\left(\overline{\bar{S}}\left(t_{i}\right)-\overline{\bar{S}}\left(t_{j}\right)\right):\left(\overline{\bar{S}}\left(t_{i}\right)-\overline{\bar{S}}\left(t_{j}\right)\right)}\right\}
$$

whereas $J_{1, \text { max }}$ is obtained as below:

$$
J_{1, \max }=\frac{1}{3} \max _{t \in T}\{\operatorname{tr}(\overline{\bar{\sigma}}(t))\}
$$

The fatigue criterion is parameterized by the 2 parameters $\alpha$ and $\beta$, that have to be identified from fatigue tests under 2 loading conditions, from a non defective material. To do so, fatigue specimens for torsion and tension/torsion tests have been manufactured, but the fatigue tests have not been performed yet. Thus, a couple of values identified at $N=5 \times 10^{6}$ cycles for a defect-free cast $\mathrm{T} 6 \mathrm{AlSi} 7 \mathrm{Mg} 0.6$ from the literature was used for the coefficients $\alpha$ and $\beta[17,18]$, in a first approach.

In order to account for the local stress gradient when assessing defect criticity, a non local approach is used. Since it is easily applicable for complex-shaped defects, the volume based method proposed by Taylor [10] is employed: for each integration point, the stresses $\tau_{o c t, a}$ and $J_{1, \max }$ are averaged over a volume $V_{r}$ corresponding to a sphere of radius $r$. The criterion is then reached when the averaged equivalent stress $\left\langle\sigma_{C R}\right\rangle=\left\langle\tau_{o c t, a}\right\rangle+\alpha\left\langle J_{1, \max }\right\rangle$ equals $\beta$. The value for parameter $r$ was arbitrarily set to $10 \mu \mathrm{m}$ to limit computational cost, and will be properly identified once the parameters $\alpha$ and $\beta$ for Crossland criterion have been determined. 


\section{Results and Discussions}

\subsection{Fatigue tests}

Figure 5a shows $\mathrm{S}-\mathrm{N}$ curve from the fatigue tests on NDP specimens. The fatigue resistance at $2 \times 10^{6}$ was assessed using staircase method, and was found to be $\sigma_{\max }=152 \mathrm{MPa} \pm 8 \mathrm{MPa}$. The analysis of the fracture surfaces revealed that the critical fatigue cracks systematically initiate from subsurface defects (figure 5b), whose distance to the specimen surface is between $20 \mu \mathrm{m}$ and $100 \mu \mathrm{m}$. One can note the important scatter of the fatigue lives. The latter can be attributed to the variability of the defects that are responsible for fatigue damage, whose sizes $\sqrt{\text { area }}$ vary from $30 \mu \mathrm{m}$ to $130 \mu \mathrm{m}$.

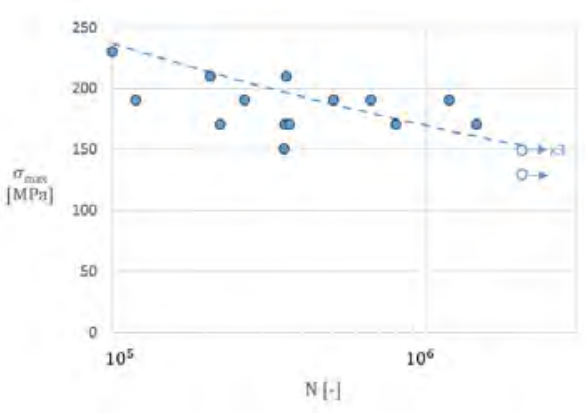

(a) S-N curve

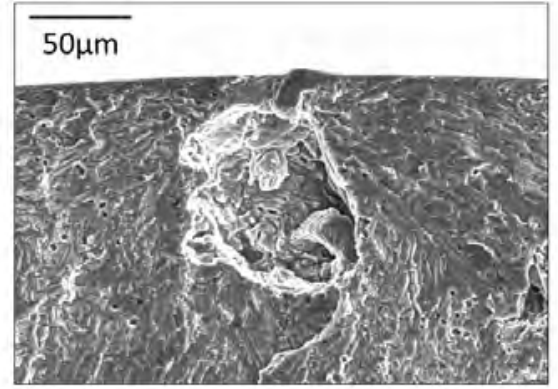

(b) SEM observation of a fracture surface - Defect of size $\sqrt{\text { area }}=98 \mu \mathrm{m}$ in the initiation zone

Figure 5: Fatigue test results: S-N curve and SEM observations of the fracture surfaces

\subsection{Analysis of defect criticity}

Finite-element calculations have been performed on both natural and artificial defect geometries. Figure 6 shows 3D views of the results related a natural defect, displaying the field of

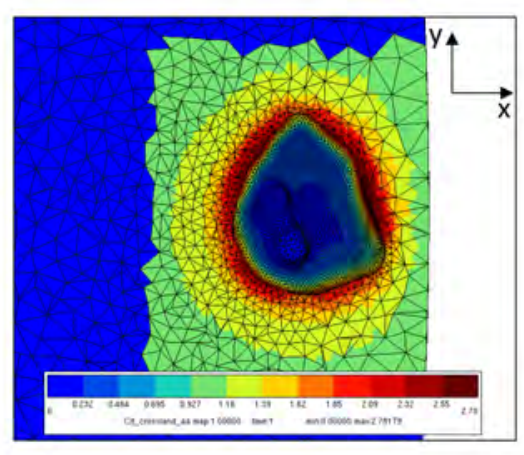

(a)

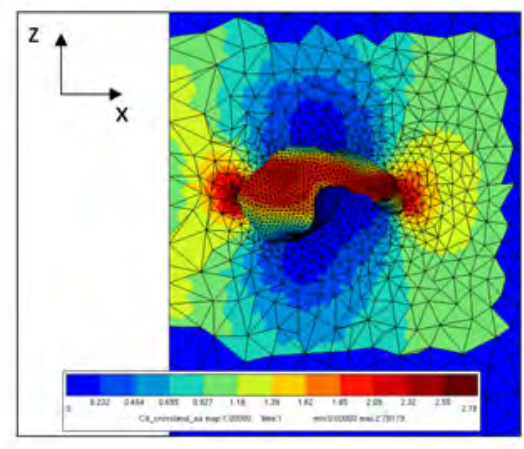

(b)

Figure 6: Views of the hazard factors $C_{d}=\left\langle\sigma_{C R}\right\rangle / \beta$ for a natural defect 
the hazard factor $C_{d}=\left\langle\sigma_{C R}\right\rangle / \beta$ in the vicinity of the defect. Even if the radius of the sphere used to average the stresses is still to be identified to build a proper Fatigue Indicator Parameter (FIP) $\left\langle\sigma_{C R}\right\rangle$, one can note that the fatigue criterion is significantly exceeded all around the defect. Thus, it can be assumed that the literature values for $\alpha$ and $\beta$ that were used for Crossland criterion are non-conservative. In what follows, the value of $\beta$ was modified in order to reduce the gap between the threshold and the calculated stress $\left\langle\sigma_{C R}\right\rangle$ for natural defects, while keeping the same value for the slope $\alpha$. The threshold so obtained is for illustrative purpose only, and aims to facilitate the comparison between the results related to natural and artificial defects. However, it is important to highlight that it cannot be employed to predict fatigue strength yet. It should be noted that the specimens containing the defects used for FE calculations have not been subjected to fatigue tests yet, and that it was not possible to use the results to adjust the value of $\beta$.

Figure 7 shows the mechanical responses in terms of $\left\langle\tau_{\text {oct, } a}\right\rangle-\left\langle J_{1, \text { max }}\right\rangle$ obtained from simulations for both natural defects (figure 7a) and artificial defects (figure 7b). The size and position with respect to the surface of each defect are also indicated. For artificial defects, the applied stress $\sigma_{\max }$ had to be lowered to $65 \mathrm{MPa}$ so that the gap between the threshold and the calculated FIP $\left\langle\sigma_{C R}\right\rangle$ is approximately the same as for natural defects. This means that a significant decrease of the fatigue resistance is expected for the fatigue specimens containing artificial defects. For natural defects, it can be observed that the criticity varies significantly from one defect to another, which is consistent with the scattering of the experimental results exhibited in 3.1. One can also note that the criticity is correlated with the defect size $\sqrt{\text { area }}$. For artificial defects, the mechanical responses are found to be more reproducible than for natural defects. Besides, it is worth noting that Defect 1 , which corresponds to the most critical defect in figure $7 \mathrm{~b}$, was closer to the surface than the other defects. Thus, these results suggest that scattering is likely to be reduced when testing specimens with CAD artificial defects.

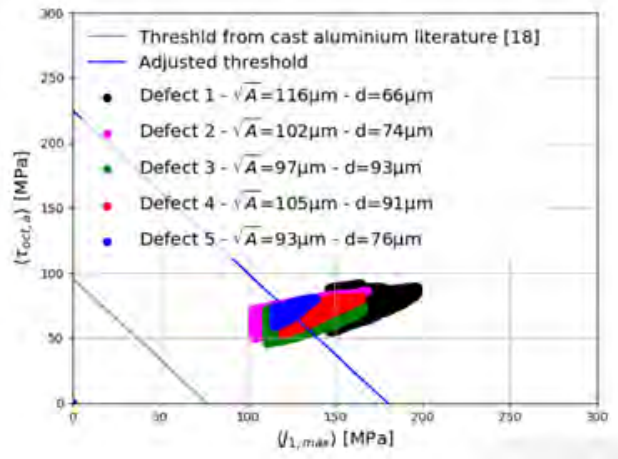

(a) Natural defects $-\sigma_{\max }=152 \mathrm{MPa}$

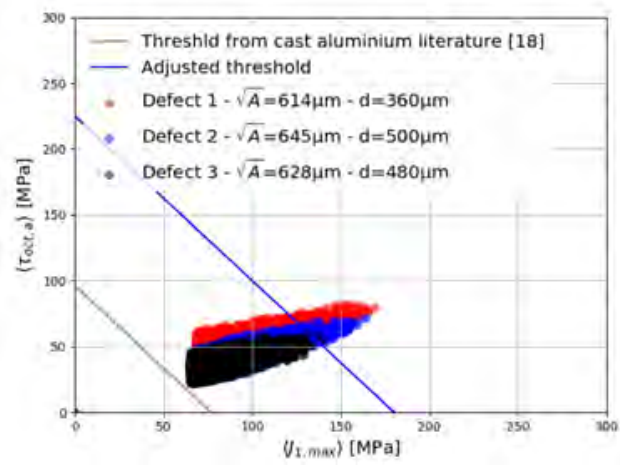

(b) Artificial defects $-\sigma_{\max }=65 \mathrm{MPa}$

Figure 7: Mechanical responses in terms of $\left\langle\tau_{o c t, a}\right\rangle-\left\langle J_{1, \text { max }}\right\rangle$ obtained from simulations

\section{Conclusion}

This paper detailed a method to evaluate the influence of defects on the fatigue behavior of an Al-Si alloy additively manufactured by SLM. A multiaxial fatigue criterion was used from FE simulations, accounting for the real defect geometries that were prior obtained with X-ray 
tomography observations of fatigue specimens. Besides, artificial defects of controlled sizes and positions were successfully introduced in some fatigue specimens. In the future, it is aimed to use such defects to investigate the influence of the defect position on the fatigue criticity, by discriminating the influence of defect size from the influence of defect position. The first simulation results showed that the mechanical responses are more reproducible for these defects compared to natural defects. At the present time, further experimental work is being carried out, firstly to identify a proper non-local fatigue criterion based on Taylor approach, and secondly to investigate defect sensitivity combining fatigue test results with numerical analysis.

\section{References}

[1] E. Olakanmi, R. Cochrane, K. Dalgarno, A review on selective laser sintering/melting (SLS/SLM) of aluminium alloy powders: Processing, microstructure, and properties, Progress in Materials Science 74, 401 (2015)

[2] S. Siddique, M. Imran, F. Walther, Very high cycle fatigue and fatigue crack propagation behavior of selective laser melted AlSi12 alloy, International Journal of Fatigue 94, 246 (2017)

[3] K. Yang, P. Rometsch, T. Jarvis, J. Rao, S. Cao, C. Davies, X. Wu, Porosity formation mechanisms and fatigue response in Al-Si-Mg alloys made by selective laser melting, Materials Science and Engineering: A 712, 166 (2018)

[4] S. Romano, A. Brückner-Foit, A. Brandão, J. Gumpinger, T. Ghidini, S. Beretta, Fatigue properties of AlSilOmg obtained by additive manufacturing: Defect-based modelling and prediction of fatigue strength, Engineering Fracture Mechanics 187, 165 (2018)

[5] Y. Murakami, Metal Fatigue: Effects of Small Defects and Nonmetallic Inclusions, elsevier edn. (2002)

[6] T. Kitagawa, Applicability of fracture mechanics to very small cracks, ASM Proceedings of 2nd international conference on mechanical Metalspark pp. 627-631 (1976)

[7] M. El Haddad, T. Topper, K. Smith, Prediction of non propagating cracks, Engineering Fracture Mechanics 11, 573 (1979)

[8] V. Le, Ph.D. thesis, Ecole Nationale Supérieure des Arts et Métiers (2016)

[9] K.J. Miller, The Short Crack Problem, Fatigue \& Fracture of Engineering Materials and Structures 5, 223 (1982)

[10] D. Taylor, Geometrical effects in fatigue: a unifying theoretical model, International Journal of Fatigue 21, 413 (1999)

[11] D. Taylor, Prediction of fatigue failure location on a component using a critical distance method, International Journal of Fatigue 22, 735 (2000)

[12] Y. Nadot, T. Billaudeau, Multiaxial fatigue limit criterion for defective materials, Engineering Fracture Mechanics 73, 112 (2006)

[13] B. Crossland, Effect of large hydrostatic pressures on the torsional fatigue strength of fan alloy steel, in Proceedings of the international conference on fatigue of metals (London and New York, 1956), pp. 138-149

[14] S. Romano, A. Brandão, J. Gumpinger, M. Gschweitl, S. Beretta, Qualification of AM parts: Extreme value statistics applied to tomographic measurements, Materials \& Design 131, 32 (2017)

[15] J.Y. Buffière, S. Savelli, P. Jouneau, E. Maire, R. Fougères, Experimental study of porosity and its relation to fatigue mechanisms of model Al-Si7-Mg0.3 cast Al alloys, Materials Science and Engineering: A 316, 115 (2001) 
[16] I. Serrano-Munoz, Ph.D. thesis, Institut National des Sciences Appliquées de Lyon (2014)

[17] P. Mu, Y. Nadot, C. Nadot-Martin, A. Chabod, I. Serrano-Munoz, C. Verdu, Influence of casting defects on the fatigue behavior of cast aluminum AS7g06-T6, International Journal of Fatigue 63, 97 (2014)

[18] P. Mu, Y. Nadot, I. Serrano-Munoz, A. Chabod, Multiaxial fatigue design of cast parts: Influence of complex defect on cast AS7g06-T6, Engineering Fracture Mechanics 131, 315 (2014) 